\title{
AUTOMATED SETUP FOR NON-TACTILE HIGH-PRECISION MEASUREMENTS OF ROUNDNESS AND CYLINDRICITY USING TWO LASER INTERFEROMETERS
}

\author{
Michael Kühnel, Uwe Gerhardt, Vinzenz Ullmann, Eberhard Manske \\ Ilmenau University of Technology, Institute for Process Measurement and Sensor Technology
}

\begin{abstract}
An automated setup for non-tactile high-precision measurements of roundness and cylindricity of ring gauges is presented in this paper. The aim is to overcome classical problems of tactile and radial roundness measurements such as the error influences of the used rotary table and the work piece alignment and thus to increase the accuracy and reduce the measurement time.

To achieve those aims a double interferometer concept was chosen and combined with a measurement system for the work piece alignment, a high precision rotary table and an automated four-axes adjustment unit. The main alignment errors of the work pieces (e.g. ring gauges) such as eccentricity and tilting are either suppressed or directly detected and consequently reduced by the automated four-axes adjustment unit. Due to the non-tactile measurement concept and the contactless energy supply of the fouraxes adjustment unit, the radial run of the rotary table is not affected.
\end{abstract}

Index Terms - roundness and cylindricity measurement, non-tactile, interferometer, automated, high-precision

\section{INTRODUCTION}

Today, requirements for form and positional tolerance measurements of work pieces in the metal-working industry are growing obviously. Established measurement techniques are not able to comply all of these demands. For example in the automobile industry ring gauges with roundness and cylindricity deviations of less than $100 \mathrm{~nm}$ are required. Thus, measurement systems with an uncertainty of less than 10 nanometres are desperately needed for the fabrication process of these ring gauges. Furthermore, decreased measurement time and an increased degree of automation is desirable.

\section{STATE OF THE ART}

Basically, the determination of roundness and cylindricity is carried out using rotary tables. Therefore the work piece (e.g. a ring gauge) is rotated and its radial roundness deviation $r(\varphi)$ is measured with respect to this rotation axis, see Fig. 1. The rotation axis of the table has to be congruent with the middle axis of the work piece. Therefore, the work piece must be aligned exactly with respect to the rotation axis of the spindle prior to each measurement. Established measurement systems utilize callipers based on an inductive measurement principle [1], [2].

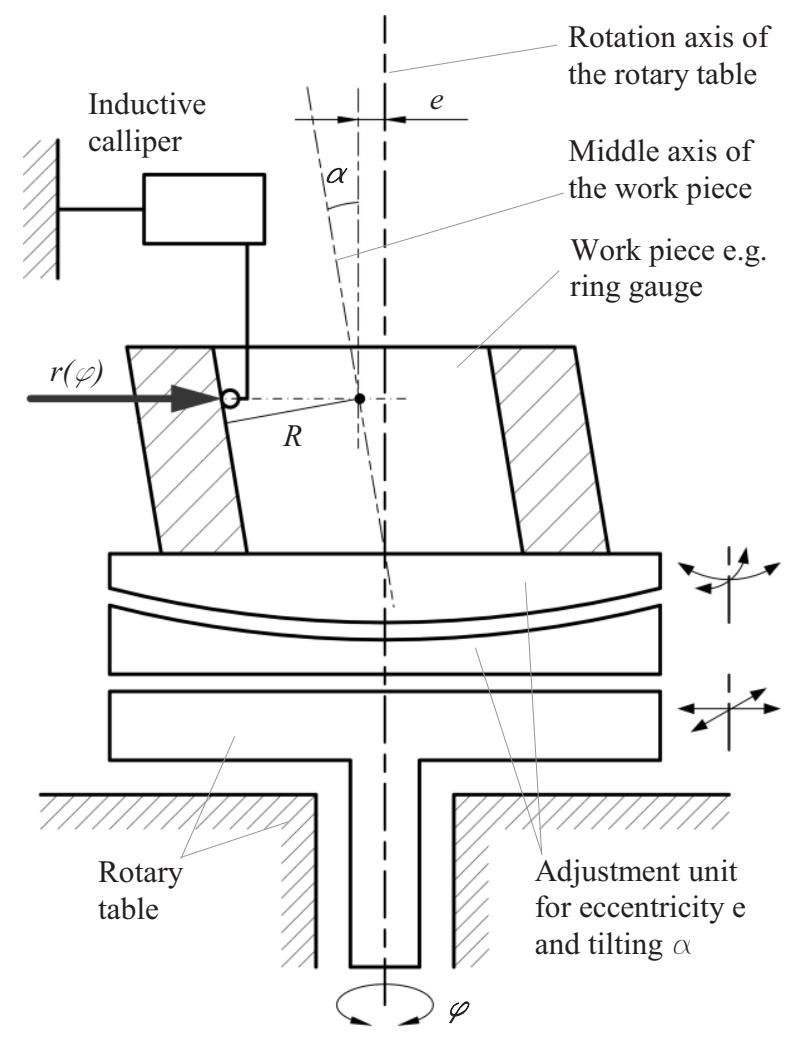

Fig. 1: principle of the state of the art of roundness measurement

The characteristics of these systems are a measuring range of a few micrometres at a resolution of 1 - 2 nanometres and a linearity error of less than $10 \mathrm{~nm}$ [3]. Due to this tactile technique additional influences to the concentric run-out and the wobble of the rotary table are generated and the measurement velocity is limited to 2 millimetres per second. Due to the small measurement range of the tactile calliper, the work piece must be aligned manually prior to the first measurement to prevent damages of the calliper. In contrast to that, the measurement velocity can be increased by the use of non-tactile optical measurement methods. The Bosch concern offers a measuring device based on an interferometrical stylus 
system for roundness and cylindricity deviations with a depth resolution of $1 \mathrm{~nm}$ [4]. The utilization of low coherent light limits the measuring range of these form deviations down to some micrometres. However, there is a common problem for both measurement systems described above. Alignment errors of the work piece axis with respect to the spindle axis, such as eccentricity $e$ and tilting $\alpha$, as well as spindle errors, as for instance concentric run-out deviations and wobbling, have a direct influence to the measured radius deviation and cause a significant contribution to measurement uncertainty. The needed alignment procedure is costly in terms of time.

\section{APPLIED PRINCIPLE OF MEASUREMENT AND FORMER INVESTIGATIONS}

To overcome the disadvantages mentioned above, a novel non-tactile interferometric measuring technique for roundness and cylindricity of ring gauges with a diameter of $D=10-300 \mathrm{~mm}$ was proposed and investigated in a former publication [5]. A double interferometer setup was chosen to suppress error influences on the measurements such as the eccentricity or radial run-out errors of the spindle, see Fig. 2. Therefore two $180^{\circ}$ shifted radial roundness deviations $r(\varphi)$ and $r\left(\varphi+180^{\circ}\right)$ are measured by the interferometers. The sum of these two radial signals represents the diameter variation $d(\varphi)$ of the ring gauge. In this sum signal of both interferometer axes, the eccentricity error, the wobble and concentric runout of the spindle and mechanical vibrations are suppressed very well. Another advantage of the double beam setup arises from the difference signal of the two interferometer axis. It represents the fourfold eccentricity and can thus be used for a fast and exact

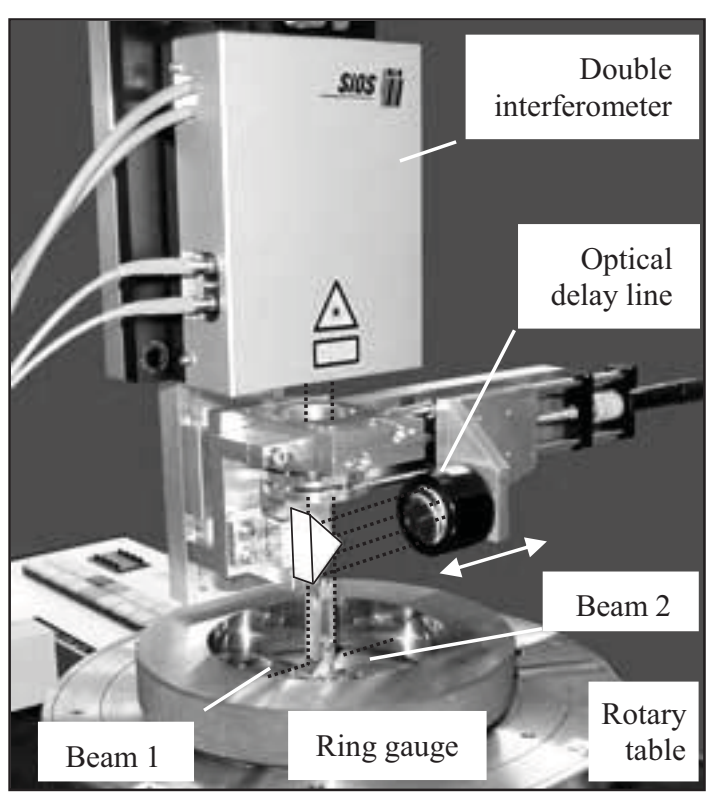

Fig. 2: double beam interferometer described in [5] alignment of the work piece on the rotary table.
The applied plane mirror interferometers were developed at the TU Ilmenau in the past [6] and are manufactured by the SIOS Meßtechnik $\mathrm{GmbH}$ company. They permit a resolution of $0.1 \mathrm{~nm}$, a nearly unlimited measurement range and velocity and nonlinearities of $<2 \mathrm{~nm}$. Due to the cylindrical form of the ring gauges the interferometer beams were focused on the ring surface using a cylindrical lens $(f=250 \mathrm{~mm})$. To adapt the focus to ring diameters from 10 to $300 \mathrm{~mm}$ an optical delay line was used. Thereto the corner cube reflector must be shifted by $145 \mathrm{~mm}$.

\subsection{Results}

An error influence on the sum signal of just $<15 \mathrm{~nm}$ based on an eccentricity e $\leq 40 \mu \mathrm{m}$ was achieved by measurements of a ring gauge with a radius $\mathrm{R}=50 \mathrm{~mm}$, see Fig.3.

Furthermore it was shown that distortions in the single axis signals introduced by mechanical vibrations in

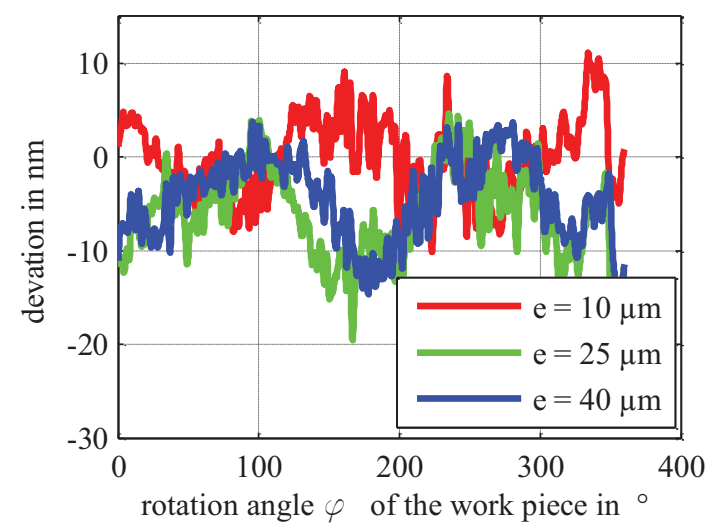

Fig. 3: error influence of the eccentricity $e$ on roundness measurements of a ring gauge $(R=50 \mathrm{~mm})$

the order of $1 \mu \mathrm{m}$ can be suppressed in the sum signal completely and influences of the spindle run-out error can be minimized. A repeatability of 4-10 nm was achieved in different scans.

The results prove a very good suitability of the double interferometer setup for roundness measurements of ring gauges even at high eccentricities.

\subsection{Problems}

Besides the promising results, the performed investigations also showed potential for further improvements.

\subsubsection{Tilting of the work piece}

In general, tilting of the work piece generates measurement deviations. This is a common problem for the measurement setups based on the state of the art as well for the presented double interferometer setup. The deviation of a radial measurement can be described as follows (cf. Fig. 4): 


$$
\Delta_{\text {till } 1}=R\left(\frac{1}{\cos \alpha}-1\right)
$$

If the roundness measurements are performed by the described plane mirror interferometers an additional deviation arises due to the tilting of the reflection surface $\alpha$, whereas $L_{\text {beam }}$ is the length of the interferometer beam [6]:

$$
\Delta_{\text {tilt } 2}=L_{\text {beam }}(\cos (2 \alpha)-1)
$$

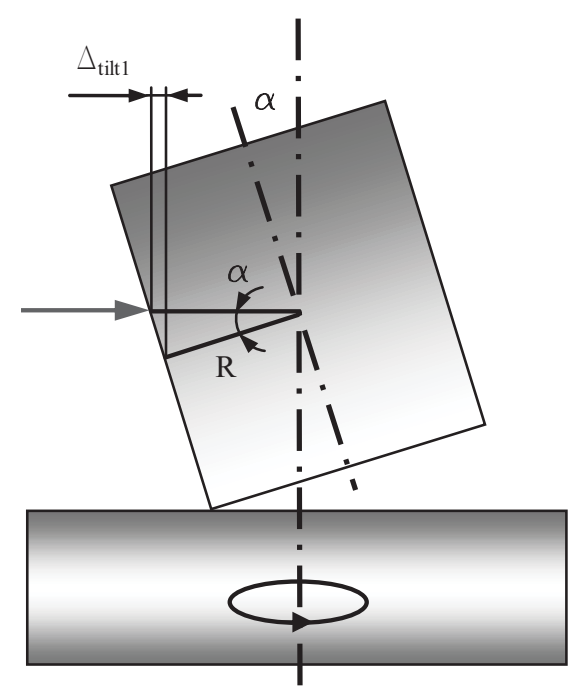

Fig. 4: error influence of tilting $\alpha$ on the roundness measurement

If a diameter measurement of the roundness deviation is carried out, the given deviation $\Delta_{\text {tilt }}$ and $\Delta_{\text {tilt } 2}$ doubles. The applied interferometers could accept a tilt of the reflecting surface $\alpha \leq 1^{\prime}$. Based on this angle, a ring gauge radius of $R=50 \mathrm{~mm}$ and a length of the interferometer beams of $L_{\text {beam }}=350 \mathrm{~mm}$ as it is applied in the setup described above, the measurement deviations of each of the two radial signals $r(\varphi)$ and $r\left(\varphi+180^{\circ}\right)$ result in:

$$
\Delta_{\text {tilt } 1}=5 \mathrm{~nm} \quad \Delta_{\text {tilt } 2}=-60 \mathrm{~nm}
$$

The complete measurement deviation for the diameter variation $d(\varphi)$ due to a tilting of the work piece can be given as:

$$
\Delta_{\text {tilt }}=2 \cdot\left(\Delta_{\text {tilt } 1}+\Delta_{\text {tilt } 2}\right)
$$

Therefore, the contribution of tilting to the measurement deviation of the presented setup would result in $\Delta_{\text {tilt }}=-110 \mathrm{~nm}$ for the given values of $R$, $L_{\text {beam }}$ and $\alpha$. Thus, tilting must be considered for the defined goal of measuring roundness deviations in the range of $100 \mathrm{~nm}$. Usually tilting could be reduced iteratively by adjustments based on a determination of the eccentricity in different levels of the work piece and is thus a very time consuming procedure. It is obvious that less time consuming methods are highly desirable. The applied optical measurement technique offers a promising approach for a fast and reliable detection of the work piece tilting: Work piece tilting can be determined by the measurement of the deflection of the reflected laser beams. Additionally a reduction of the interferometer beam length $L_{\text {beam }}$ will minimize this error influence (cf. Equ. 2).

\subsubsection{Beam focusing}

In further experiments ring gauges with small radii $(R<25 \mathrm{~mm})$ caused a significant widening of the reflected laser beam. Those distortions avoided proper functioning of the interferometers. A widening of the reflected laser beams arises due to the spherical shape of the reflecting ring surface although the beams are focused by a single lens $(f=250 \mathrm{~mm})$. The reason for this is the residual waist of the beam in the focal plane. The magnitude of the beam waist is a property of the Gaussian beam and can be reduced using smaller focal lengths $f$. Thus, a widening of the reflected beams can be minimized applying smaller focal lengths, especially for ring gauges with small radii.

\section{NEW SETUP}

Due to the encouraging results and the potential for improvements described above, a complete measuring setup based on the described double interferometer concept was designed.

\subsection{Principle}

The new setup consists of seven main structural components: a new double interferometer setup, a beam focusing unit, a measuring unit for work piece tilting and eccentricity, a rotary table, a stacked on four-axis adjustment unit for a fast, automatic and precise alignment of the work piece, a rack carrying the whole setup and a controlling unit. The basic principle of the setup is shown in Fig 5. The measurement procedure can be described as follows:

The ring gauge is applied on the four-axes adjustment unit, the focus is set dependent on the ring radius. Then the ring must be pre-adjusted manually to hit the measurement range of the tilting measurement unit and to provide an interferometer signal. Due to the use of laser beams the manual adjustment is very easy compared to tactile methods. The work piece must just be aligned in that way, that the reflected laser beam is roughly congruent with the beams that are send out by the interferometers.

Subsequently the work piece will be aligned automatically and with high precision by the four-axes adjustment unit. The four-axes adjustment unit works based on the measured alignment information of the tilting measurement unit and the difference signal of the two interferometers (c.f. section 3). 
Finally the roundness and cylindricity measurements can be performed at different levels of the ring gauge.

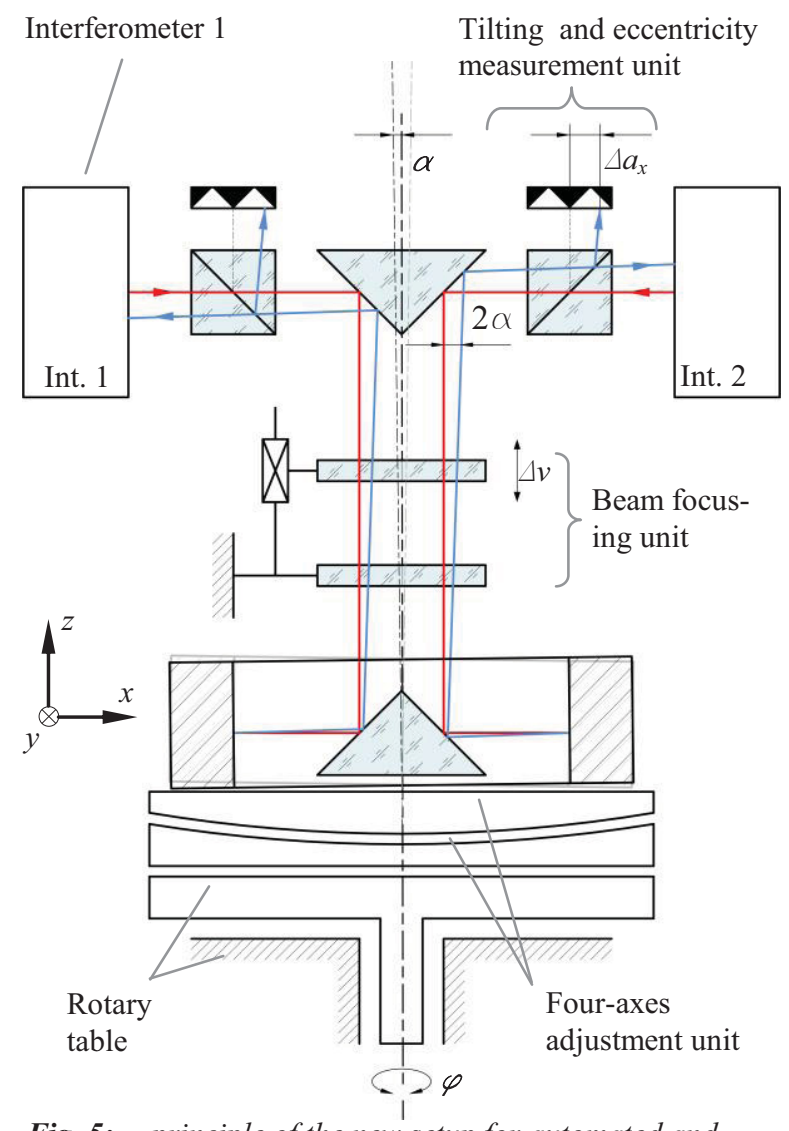

Fig. 5: $\quad$ principle of the new setup for automated and non-tactile roundness and cylindricity measurements of ring gauges

\subsection{Main structural components}

In the following, the seven main structural components are described explicitly.

\subsubsection{Interferometer}

The interferometer setup consists of two SIOS-PlaneMirror-Interferometers (SP 2000) [6] including adjustment elements for the direction and position of each interferometer beam. The resolution of the interferometers is $0.1 \mathrm{~nm}$, at a measurement range of up to $2000 \mathrm{~mm}$. The applied He-Ne-lasers ensure a frequency stability of $2 \cdot 10^{-8}$ at a wave length of $632.8 \mathrm{~nm}$. Systematic influences of the temperature, the humidity and the air pressure on the wavelength are corrected. An interferometer beam guiding system is needed to measure the two $180^{\circ}$ shifted radial roundness deviations of ring gauges. This is realized by two adjustable $90^{\circ}$ prisms. Interferometer 1 measures the radial roundness deviation $\mathrm{r}(\varphi)$ and interferometer 2 measures $r(\varphi+180)$ at the same time. The interferometer beams are focused on the ring surface by the beam focusing unit.

\subsubsection{Beam focusing unit}

A new beam focusing unit was developed to meet the following requirements: smaller focal lengths for the measurement of ring gauges with small radii, the focus must be adaptable to ring gauges with diameters from $D=10-300 \mathrm{~mm}$, small size of the system. To meet all these requirements a motor driven zoom lens containing a cylindrical converging lens $\left(H_{l} H_{l}{ }^{\prime}\right.$ with $\left.f_{1}=50 \mathrm{~mm}\right)$ and a cylindrical diverging lens $\left(\mathrm{H}_{2} \mathrm{H}_{2}{ }^{\prime}\right.$ with $f_{2}=-50 \mathrm{~mm}$ ) was developed. The optical scheme of the zoom lens is shown in Fig. 6.

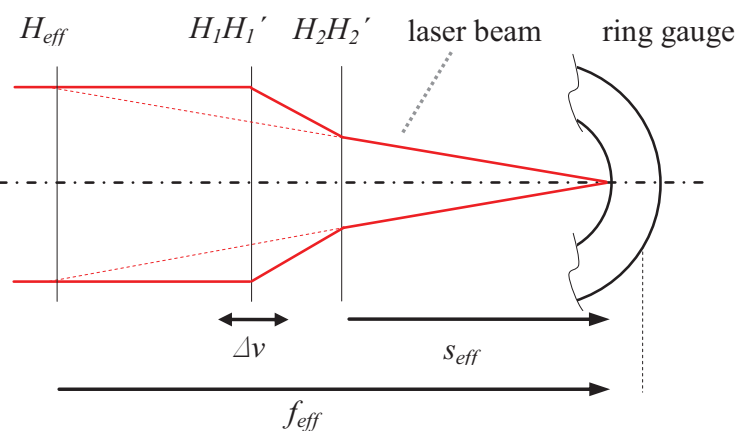

Fig.6: $\quad$ principle of the beam focusing unit

By shifting the converging lens $\left(H_{1} H_{l}{ }^{\prime}\right)$ by $\Delta v=12 \mathrm{~mm}$, the back focal length $s_{\text {eff }}$ can be adjusted in the range of $s_{\text {eff }}=70-215 \mathrm{~mm}$. Thus, the focus can be adapted to ring gauges with radii from $R=5-150 \mathrm{~mm}$ at a minimum distance of $70 \mathrm{~mm}$ between the diverging lens $\left(\mathrm{H}_{2} \mathrm{H}_{2}\right)$ and the ring surface. The corresponding effective focal length for this zoom lens is $f_{\text {eff }}=120-280 \mathrm{~mm}$. Ring gauges with a radius of $R=5 \mathrm{~mm}$ are measured at an effective focal length of $f_{\text {eff }}=120 \mathrm{~mm}$ and ring gauges with a radius of $R=150 \mathrm{~mm}$ are measured at $f_{\text {eff }}=280 \mathrm{~mm}$. The functionality of the lens system was tested based on different ring gauges. By using a ring gauge with a radius of $R=10 \mathrm{~mm}$ the widening of the reflected laser beam was in the range of $30 \%$ and a proper function of the interferometers was observed. This is a huge improvement compared to the old single lens $(f=250 \mathrm{~mm})$ where the beam widening was in the range of $300 \%$ and no evaluable interferometer signal was achieved.

Investigations showed that the position of the converging lens must be adjusted with an uncertainty of less than $\Delta a<0.5 \mathrm{~mm}$ to ensure a sufficient focusing of the interferometer beams. This could be securely achieved by the used linear motor stage which offers a position resolution of $<2 \mu \mathrm{m}$.

In contrast to the old system, the new beam focusing unit allows a focusing of the interferometer beams to ring gauges with a diameter of $D=10-300 \mathrm{~mm}$ without limiting a proper functioning of the interferometers. Furthermore the new system is obviously smaller than the single lens system with the optical delay line. 


\subsubsection{Tilting and eccentricity measurement}

The tilting and eccentricity measurement system consists of a beam splitter and a quadrant photodiode with a sensitive area of $5 \times 5 \mathrm{~mm}$. If the ring gauge tilts by $\alpha$, the reflected laser beams (blue) tilt by $2 \alpha$, see Fig. 5. Dependent on the geometrical path length $L_{\text {beam }}$ of the reflected beams, the deflection $\Delta a_{x}$ of the beams (in direction of the x-axis, c.f. Fig. 5) is detected by the quadrant diodes. The tilting angle of the work piece can be determined based on Equ. 4:

$$
\tan (2 \alpha)=\frac{\Delta a_{x}}{L_{\text {beam }}}
$$

For investigations on the tilting measurement principle a beam splitter and a quadrant diode was implemented in the old system that was described in section 3. In the first step the resolution and the repeatability of $\Delta a_{x}$ due to beam tilting was observed using a tilting mirror instead of a ring gauge. The length of the reflected beam was $L_{\text {beam }}=350 \mathrm{~mm}$. The repeatability of the deflection measurement was determined to be $\Delta a_{x}=\leq \pm 4 \mu \mathrm{m}$. Consequently, based on Equ.4, a repeatability of the tilting angle $\alpha$ of $1.2^{\prime \prime}$ is achieved with the system. To prove the principle, tilting measurements of a rotating work piece were carried out in the next step. Thereto a ring gauge $(R=50 \mathrm{~mm})$ was deposited on the rotary table and the tilting angle $\alpha$ was reduced successively based on the signal of the quadrant diode. Generally, a tilting of the work piece generates a sinusoidal deflection signal $\Delta a_{x}(\varphi)$ when the work piece is rotated by the rotary table. The magnitude of the sinusoidal signal represents the tilting angle $\alpha$. In Fig. 7 five measurements of the work piece tilting $\alpha(\varphi)$ are presented.

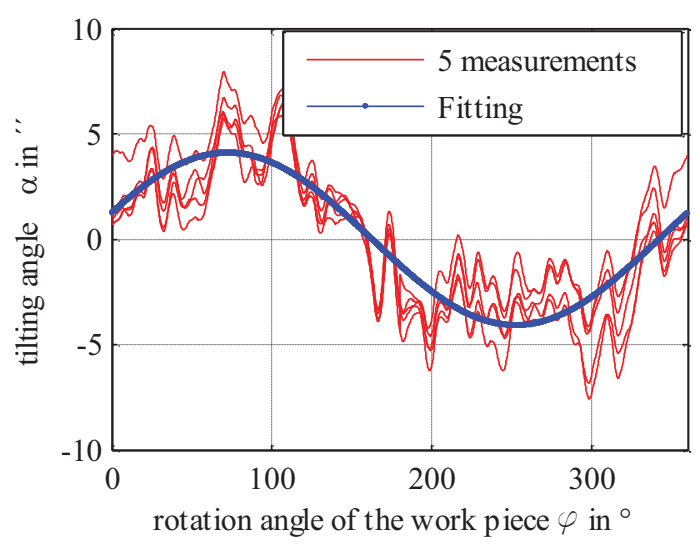

Fig. 7: $\quad$ measurements of the work piece tilting

A tilting angle of $\alpha=4 "$ ( $\left(\triangleq \Delta a_{x}=13.5 \mu \mathrm{m}\right)$ could be resolved clearly. The repeatable deviations from the ideal sinus signal are expected to be caused by the wobbling of the rotary table. Based on this considerations, a useful resolvable beam deflection of $\Delta a_{x, \min }=10 \mu \mathrm{m}$ is assumed in the following.
For the new designed system (c.f. Fig. 5) the beam splitter and the quadrant diodes are in included in the interferometer housing. The minimal length of the reflected beam is $L_{\text {beam }}=185 \mathrm{~mm}$ for measurements of ring gauges with $R=5 \mathrm{~mm}$. Based on $\Delta a_{x, \min }=10 \mu \mathrm{m}$ a tilting angle of $\alpha_{\min }=6^{\prime \prime}$ will be resolvable smoothly (c.f. Equ. 4). A tilting angle of $6 "$ would generate a measurement deviation of the roundness signal of $\Delta_{\text {tilt }}=-0.3 \mathrm{~nm}$ (c.f. Equ. 1, Equ. 2, Equ. 3). Thus, based on the presented tilting measurement system the influence of tilting on the roundness measurements can be reduced to a negligible level. This requires an adjustment unit that is able to set the tilting angle $\alpha$ with a resolution of some arc seconds.

The measurement range of the tilting measurement system is dependent on the radius of the laser beam $(\sim 1 \mathrm{~mm})$ since each of the four quadrants must be illuminated. With this maximum deflection $\Delta a_{x, \max }=1 \mathrm{~mm}$ and the length of the reflected beam $L_{\text {beam }}$, the maximum measurement range for work piece tilting can be given as (c.f. Equ. 4): $\alpha_{\max }=10^{\prime}$.

Furthermore, the work piece eccentricity $e$ can be detected by the quadrant diode. An eccentricity $e$ of the ring gauge generates a deflection of the laser beams $\Delta a_{y}$ in the $y$-direction at the quadrant diode, see Fig. 8.

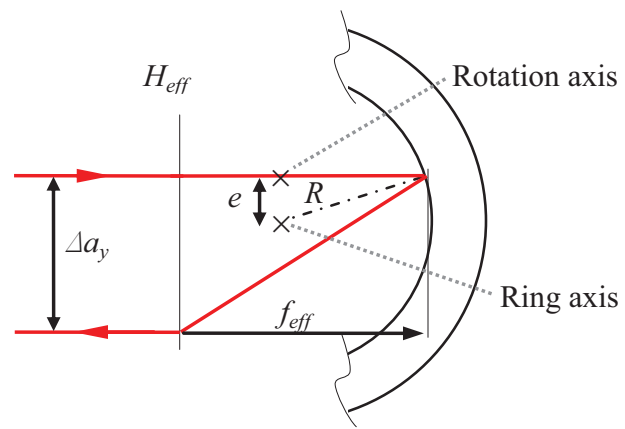

Fig. 8: principle of eccentricity detection with the four quadrant diode

The deflection is dependent on the radius $R$ of the ring gauge, the focus length $f_{\text {eff }}$ and the eccentricity $e$ and can be estimated as follows:

$$
\Delta a_{y}=\frac{2 \cdot e \cdot f_{e f f}}{R}
$$

When the work piece is rotated by the rotary table, the quadrant diode will detect a sinusoidal deflection signal $\Delta a_{y}(\varphi)$. The magnitude of this signal corresponds to the eccentricity. Assuming a useful resolution of $10 \mu \mathrm{m}$ (see above) a minimal eccentricity of $e \approx 0.4 \mu \mathrm{m}$ is detectable for Ring gauges with a radius of $R=10 \mathrm{~mm}\left(f_{\text {eff }}=125 \mathrm{~mm}\right)$. For bigger radii $R$, the detectable eccentricity increases respectively. Besides the difference signal of the interferometers, the deflection measurement 
using the quadrant diodes offers an additional opportunity for a detection of the eccentricity. It can be used for pre-adjustments as long as no interferometer signal is available due to not congruent laser beams.

\subsubsection{Rotary Table}

The used rotary table is an UPR-160 AIR Ultra Precision Rotation Stage made by miCos GmbH. It mainly consists of an air bearing and a motor. The wobble of the bearing is given as $\pm 0.26^{\prime \prime}$, the concentric run out is given as $\pm 0.1 \mu \mathrm{m}$. Due to this high accuracy it is perfectly suitable for the means of high precision roundness measurement tasks. A $35 \mathrm{~mm}$ centre hole in the rotary table offers the option to include a system to transfer electrical energy and information form the static part to the rotating and thus to the stacked on four-axes adjustment unit.

\subsubsection{Four-axes adjustment unit}

For the automatic alignment of the ring gauge axis with respect to the axis of the rotary table the fouraxes adjustment unit was developed and is stacked on the top of the rotary table, c.f. Fig.5.

The adjustment unit consist of two stacked motor driven linear axes for the adjustment of the eccentricity in $\mathrm{x}$ - and $\mathrm{y}$-direction and two motor driven tilting axes for the adjustment of the tilting whereas this tilting stage is stacked on the linear stages.

Each of the two linear stages comprises a stepper motor including a planetary gear, a screw type gear to transform the rotation in a translation and a primary and a secondary linear ball bearing. The adjustment range is designed to $\pm 2 \mathrm{~mm}$ for both, the $\mathrm{x}$ - and the $\mathrm{y}$ axis. The theoretical resolution can be given as 0.2 $\mu \mathrm{m}$.

The tilting stage basically comprises a sphere and two straight-wedge mechanisms. The upper level of the adjustment unit is supported by these three points. The work piece will later be placed on the upper level of the adjustment unit. By changing the z-level of the two straight-wedge mechanisms the upper level of the adjustment unit can be tilted around the $\mathrm{x}$ - and the $y$-axis. The centre of this tilting is defined by the centre of the sphere. The two straight-wedge mechanisms are driven by the same motor and gear combination as it is used for the linear stage. A tilting range of $\alpha= \pm 1^{\circ}$ is realized with the system. The theoretical resolution is $0.1^{\prime \prime}$.

The four-axes adjustment unit is stacked on the rotating part of the rotary table (spindle). To operate the four motors of the adjustment unit based on the measured alignment information without disturbing the run of the high precision rotary table, a noncontact transmission of energy and information between the stator and the spindle of the rotary table is essentially. Therefore an inductive transmission of the electrical energy and an optical data transfer was developed in consideration of the small installation space and was tested successfully.

To sum up, the four-axes alignment unit enables a high precision adjustment of the work piece with respect to the rotation axis. Thus, the error influences eccentricity and tilting of the work piece can be reduced to a negligible level. Due to the contactless transport of energy and information, the run of the high precision rotary table is not disturbed.

\subsubsection{Rack}

Furthermore the double interferometer must be aligned with respect to the spindle axis when the system is mounted. For that reason a rack including appropriate adjustment elements was designed. Besides this, the roundness measurements must be carried out at different levels of the ring gauges to determine the cylindricity. Thereto the interferometer must be shift able in z-direction. This is implemented by the use of a linear guiding system z-direction.

\subsubsection{Controlling unit}

All the measured data will be processed by a personal computer. This includes the alignment information of the work piece, the measured interferometer signals and the z-position of the interferometer. Furthermore the motors, including rotary table, adjustment unit and z-position of the interferometer will be driven by a computer. Thus, the setup will be completely automated.

\subsection{Complete Setup}

The complete setup for automated, non-tactile highprecision measurements of roundness and cylindricity is shown in Fig. 9.

\section{CONCLUSIONS}

A setup for automated, non-tactile high-precision measurements of roundness and cylindricity of ring gauges was presented. The setup bases on a double interferometer concept described in [5]. The classical error influences on roundness- and cylindricity measurements such as eccentricity and tilting of the work piece and wobbling of the rotary table are reduced or compensated. Due to the results of the described investigations the resulting contribution of these error influences to the measurement deviation will be in the $10 \mathrm{~nm}$ range.

Furthermore, the essential work piece alignment procedure will be timesaving compared to the wellknown tactile methods. This is achieved by a direct detection of the alignment errors tilting and eccentricity and the automated four-axes adjustment unit. Because of the non-tactile method the measurement velocity is nearly not limited. This leads to an additional time saving especially for whole cylinder scans. 
To sum up, the set is a promising approach for measurements of smallest roundness and cylindricity deviations in the $100 \mathrm{~nm}$ range.

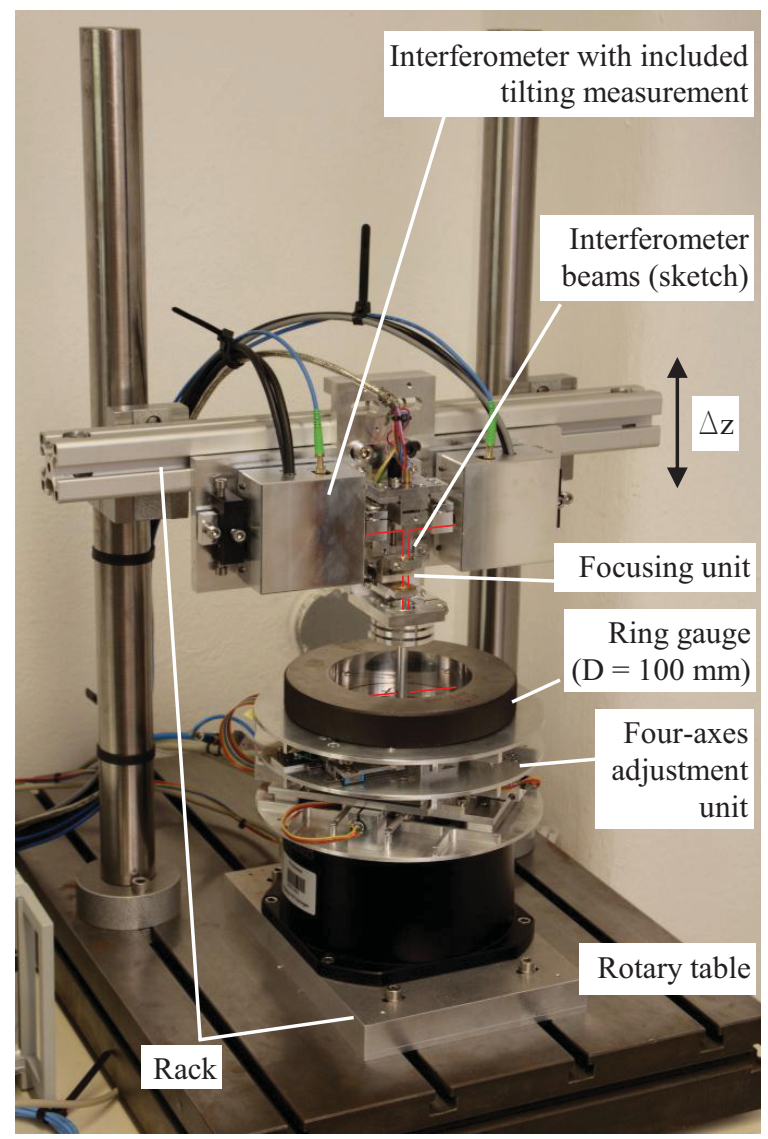

Fig. 9: complete measurement setup for automated and non-tactile roundness and cylindricity measurements of ring gauges

\section{OUTLOOK}

The setup will be put in to operation in the future. The interaction of all components will be tested and measurements and investigations on the system performance and measurement uncertainty will be carried out.

\section{ACKNOWLEDGMENTS}

Special thanks to SIOS Meßtechnik GmbH Ilmenau and Lehren- und Messgerätewerk $\mathrm{GmbH}$ Schmalkalden.

\section{REFERENCES}

[1] www.mahr.de

[2] www.taylor-hobson.com

[3] R. Thalmann, "Basics of highest accuracy roundness measurement." Simposio de Metrologia 2006 of CENAM, 25.-27.Octobre 2006, Queretaro, Mexico

[4] http://pdf.directindustry.com/pdf/mycrona/boschfms-1000-data/26866-108701.html

[5] E. Manske, G. Jäger, I. Schmidt, W. Pöschel, M. Kühnel, "High-precision estimation of circle and cylinder form deviations by means of a fibre-coupled laser interferometer arrangement" Proceedings of the $7^{\text {th }}$ international conference european society for precision engineering and nanometrology 2007

[6] H.-J. Büchner, G. Jäger, „A novel plane mirror interferometer without using corner cube reflektors" Measurement science and technology.Bristol: IOP Publ.Ltd., ISSN 0957-0233, Bd. 17 (2006) 